\title{
Références bibliographiques du dossier « La pédagogie universitaire "
}

Prunelle Charvet et Hélène Beaucher

\section{(2) OpenEdition \\ 12 Journals}

Édition électronique

URL : https://journals.openedition.org/ries/8336

DOI : 10.4000/ries.8336

ISSN : 2261-4265

Éditeur

France Education international

\section{Édition imprimée}

Date de publication : 1 avril 2019

Pagination : 135-148

ISBN : 978-2-85420-623-4

ISSN : 1254-4590

Référence électronique

Prunelle Charvet et Hélène Beaucher, «Références bibliographiques du dossier « La pédagogie universitaire » », Revue internationale d'éducation de Sèvres [En ligne], 80 | avril 2019, mis en ligne le 01 avril 2019, consulté le 24 juin 2021. URL : http://journals.openedition.org/ries/8336 ; DOI : https:// doi.org/10.4000/ries.8336 


\title{
Références bibliographiques du dossier "La pédagogie universitaire "
}

\author{
Prunelle Charvet \\ Direction générale de l'enseignement supérieur \\ et de l'insertion professionnelle \\ Hélène Beaucher \\ France Éducation international
}

La massification de l'enseignement supérieur, le développement de la mobilité internationale, la pression des classements internationaux et la généralisation du numérique sont autant de facteurs qui ont conduit à une transformation de l'enseignement et au développement de la pédagogie universitaire.

Ces dernières années, l'amélioration de l'apprentissage et de l'enseignement est devenue une priorité, non seulement pour les établissements d'enseignement supérieur mais également pour les gouvernements nationaux, l'Union européenne et le processus de Bologne. Le Communiqué d'Erevan de 2015, signé par 47 ministres européens responsables de l'enseignement supérieur et de la recherche, met en outre l'accent sur la mission « d'amélioration de la qualité et de la pertinence de l'apprentissage et de l'enseignement » que doit relever l'espace européen de l'enseignement supérieur.

Cette sélection bibliographique rassemble, dans un premier temps, des références introductives au thème de la pédagogie universitaire ainsi que des éléments de contexte international sur les grands défis auxquels l'enseignement supérieur doit faire face et qui influent sur les pratiques pédagogiques. Elle présente ensuite des rapports qui témoignent des politiques européennes et nationales en faveur de l'amélioration de l'enseignement et de l'apprentissage dans l'enseignement supérieur. Enfin, d'autres leviers d'amélioration de la pédagogie universitaire sont présentés à travers des études de cas : dispositifs et pratiques pédagogiques innovants, espaces physiques d'apprentissage, évaluation des compétences acquises et de la qualité des enseignements, technologies éducatives, développement professionnel des enseignants-chercheurs et recherche en éducation.

Parce que la pédagogie dans l'enseignement supérieur est un sujet d'étude et d'intérêt relativement récent, la sélection des références bibliographiques se limite aux publications des dix dernières années. Les titres proposés dans cette sélection complètent ceux donnés par les auteurs des articles de ce dossier 80 de la Revue internationale d'éducation de Sèvres.

Bibliographie arrêtée le 10 mai 2019. 


\title{
INTRODUCTION À LA PÉDAGOGIE UNIVERSITAIRE
}

\author{
DE KETELE Jean-Marie, "La pédagogie universitaire : un courant en plein développe- \\ ment », Revue française de pédagogie, septembre 2010, $n^{\circ}$ 172, p. 5-76 [en ligne]
}

Les analyses et les recherches sur la qualité des formations universitaires sont relativement récentes et ont vu le jour il y a trois à quatre décennies dans les domaines scientifiques les plus professionnalisants, tels que les sciences médicales. L'intérêt pour la pédagogie universitaire s'est accéléré ces dernières années sous la pression de différents facteurs : des réunions internationales importantes, comme le congrès mondial de l'enseignement supérieur organisé à Paris par l'Unesco en 1998, des événements politiques majeurs, comme la réunion de Bologne et le sommet de Lisbonne, la multiplication des centres de ressources pédagogiques au sein des universités et l'émergence d'associations internationales et nationales ayant pour but d'améliorer la qualité des enseignements universitaires. http://rfp.revues.org/2168

\section{DE KETELE Jean-Marie, HUGONNIER Bernard, PARMENTIER Philippe et COSNEFROY Laurent, Quelle excellence pour l'enseignement supérieur ?, De Boeck/ Bruxelles, 2016, 226 p. \\ La première partie de l'ouvrage cherche à définir ce qu'est l'excellence avant de s'intéresser aux missions des enseignants-chercheurs dans le contexte du processus de Bologne, à l'excellence dans le cadre du curriculum, puis à l'innovation. Enfin, elle aborde la question de l'évaluation de l'enseignement et de la recherche. La deuxième partie propose des présentations des poli- tiques d'excellence menées dans le domaine de l'enseignement supérieur dans douze pays ainsi qu'une analyse synthétique et comparative de ces textes.}

\section{LAMEUL Geneviève, LOISY Catherine (dir.), La pédagogie universitaire à l'heure du} numérique : questionnement et éclairage de la recherche, De Boeck/Bruxelles, 2014, $249 p$.

L'ouvrage s'inscrit dans un contexte marqué par les changements au sein de l'Université et par la place grandissante des technologies du numérique. Les contributions sont organisées selon deux axes. La première partie construit une trame conceptuelle, contextuelle et méthodologique pour situer, problématiser et analyser la pédagogie universitaire numérique. La seconde partie illustre par des exemples concrets des articulations entre recherche, pratiques pédagogiques intégrant le numérique, et accompagnement.

\section{STEVENTON Graham, BROUGHAN Christine et CLOUDER Lynn, Global} Perspectives on Teaching Excellence: A new era for higher education, Routledge/ Londres, 2018, 250 p.

Cet ouvrage rassemble les contributions de divers experts internationaux sur la manière dont l'enseignement est mesuré et valorisé dans l'enseignement supérieur. Il s'intéresse notamment à la mise en œuvre des cadres de qualité et des prix d'excellence et propose de développer plus largement de telles initiatives. 


\title{
CONTEXTE INTERNATIONAL
}

\author{
Massification \\ et internationalisation \\ de l'enseignement supérieur
}

CHEI : Centre for higher education internationalisation, EAIE : European association for international education et IAU - Association internationale des universités, L'internationalisation de l'enseignement supérieur, Parlement européen/Bruxelles 2015, $374 p$. [en ligne]

Cette étude cherche à comprendre ce que signifie l'internationalisation de l'enseignement supérieur dans le contexte européen et les objectifs qu'elle doit atteindre. Elle propose une vue d'ensemble des principales stratégies poursuivies à divers niveaux (mondial, européen, national, institutionnel) et s'interroge sur l'opportunité que représentent l'apprentissage numérique et la mobilité virtuelle pour remplacer les formes traditionnelles de mobilité du personnel et des étudiants. L'étude présente 17 rapports nationaux en Europe et dans le monde ainsi que des recommandations pour l'élaboration de futures politiques dans ce domaine, tant au niveau européen que national, mais également local. http://goo.gl/s8WHXO

HAZELKORN Ellen (ed.), COATES Hamish (ed.), McCORMICK Alexander C. (ed.), Research handbook on quality, performance and accountability in higher education, Edward Elgar Publishing/Cheltenham, 2018, 590 p.

Alors que l'enseignement supérieur est devenu un facteur déterminant de la compétitivité économique, les établissements sont soumis à des pressions croissantes pour démontrer leur aptitude à répondre aux besoins de la société et des individus. Les questions relatives à la qualité, à la performance et au rendement de l'enseignement supérieur sont au cœur de ces préoccupations. Mêlant de nouvelles recherches à des exemples nationaux et régionaux contextualisés du monde entier, ce manuel de recherche réunit les contributions d'experts internationaux sur les meilleures façons de comprendre, d'évaluer et d'améliorer la qualité, la performance et la responsabilité dans l'enseignement supérieur.

LIOGIER Valéri, "Près de 3 millions d'étudiants en mobilité internationale dans l'OCDE ", Note d'information " Enseignement supérieur et recherche ", juin 2016, $n^{\circ} 16.03,8 p$., [en ligne]

En 2012-2013, dans la zone OCDE, près de trois millions d'étudiants sont scolarisés en dehors de leur pays d'origine. La mobilité internationale est estimée pour la première fois pour l'ensemble des pays de cette zone. La France est le quatrième pays d'accueil, avec 230000 étudiants étrangers mobiles accueillis, derrière les États-Unis, le Royaume-Uni et l'Australie. Les étudiants chinois représentent un quart des étudiants mobiles accueillis dans l'OCDE, et l'ensemble des étudiants asiatiques en représentent la moitié.

http://bit.ly/2W7TNXN

PROCTOR Douglas (ed.), RUMBLEY Laura E. (ed.), The future agenda for internationalization in higher education: next generation insights into research, policy and practice, Routledge/Londres, 2018, 242 p., (Internationalization in higher education) 
L'internationalisation de l'enseignement supérieur, phénomène mondial, est sujet à de multiples interprétations. Les différentes contributions s'organisent en trois parties : nouveaux contextes, nouveaux sujets, nouvelles modalités. Les auteurs examinent l'état de la recherche et les pratiques méthodologiques innovantes et identifient les domaines clés pour le futur.

Union européenne, The european education report, Commission européenne/Direction générale de l'éducation et de la culture/Bruxelles, avril 2018, 99 p., (Flash Eurobarometer; 466) [en ligne]

Plus de 8000 jeunes à travers l'Union européenne (UE), âgés de 15 à 30 ans ont été interrogés sur leur expérience de mobilité et leur connaissance des langues. Ils ont également donné leur avis sur un certain nombre d'initiatives récentes de l'UE visant à améliorer les environnements d'enseignement et d'apprentissage en Europe, dans le but d'œuvrer en faveur d'un espace européen de l'éducation d'ici 2025. http://bit.ly/2LS472l

\section{ÉVOLUTION DES PUBLICS « ÉTUDIANT » ET « ENSEIGNANT »}

138 Cet article propose de creuser l'idée de l'existence d'une génération Y. La recherche porte sur

DEMOUGEOT-LEBEL Joëlle, "Enseignants-chercheurs de la Génération Y : incidence sur les pratiques pédagogiques? ", Revue internationale de pédagogie de l'enseignement supérieur, vol. 30, $n^{\circ}$ 3, 2014, 19 p. [en ligne]

des enseignants que l'on pourrait qualifier d'enseignants de la génération Y. L'auteure cherche à comprendre si, au nom de cette appartenance, leurs pratiques pédagogiques sont innovantes et reflètent les attributs technologiques que l'on associe à cette génération.

http://ripes.revues.org/883

ENDRIZZI Laure et SIBUT Florence, "Les nouveaux étudiants d'hier à aujourd'hui ", IFE/Lyon. Dossier de veille de l'ifé, $n^{\circ} 106,2015,40$ p. [en ligne]

Ce dossier s'intéresse aux expériences d'études à partir d'une exploration de la littérature scientifique récente, essentiellement française. Il s'agit en creux d'examiner les conditions de vie et d'études les plus propices à la réussite étudiante et de mettre au jour les décalages potentiels entre étudiants et enseignants en termes d'attentes et de pratiques. La première partie examine les évolutions sociodémographiques, la deuxième appréhende la complexification des parcours d'études, la troisième s'intéresse aux environnements d'études et la quatrième interroge les décalages entre étudiants et enseignants. http://goo.gl/PJTq00

FLANCHEC Alice Le et MULLENBACH Astrid, "La génération Y " [dossier], Revue internationale de psychosociologie et de gestion des comportements organisationnels, Vol. XXII, $n^{\circ}$ 53, janvier 2016, 240 p. [en ligne]

Les contributions de ce numéro s'attachent à définir les spécificités de la génération Y appelée aussi « Millenials ", " millénistes », « enfants du Millénaire », " génération Internet ", " e-génération » ou encore "Echo Boomers ", qui englobe des individus nés, pour certains, entre 1979 et 1994, pour d'autres entre 1979 et 1989 ou encore après 1980. Les auteurs s'intéressent notamment à leurs valeurs morales, à leur attitude au travail et aux conséquences managériales que cela suscite. https://goo.gl/8EY66p 


\title{
Politiques européennes \\ ET NATIONALES EN FAVEUR \\ DE L'AMÉLIORATION \\ DE L'ENSEIGNEMENT \\ ET DE L'APPRENTISSAGE \\ DANS LE SUPÉRIEUR
}

\begin{abstract}
Commission européenne, "Learning and Teaching ", dans The European Higher Education Area in 2018: Bologna Process Implementation Report, Office des publications de l'Union européenne/Luxembourg, 2018, p. 47-91 [en ligne]

À la suite du Communiqué d'Erevan de 2015 qui mettait l'accent sur la mission « d'amélioration de la qualité et de la pertinence de l'apprentissage et de l'enseignement » que devait relever l'espace européen de l'enseignement supérieur, un chapitre consacré au sujet a été créé dans le rapport de mise en œuvre du processus de Bologne de 2018. Ce nouveau chapitre examine tout d'abord la place de l'apprentissage et de l'enseignement dans les stratégies et politiques de l'enseignement supérieur. Il fournit ensuite des informations sur la mise en œuvre des crédits et des résultats d'apprentissage et sur les options d'études flexibles, en particulier les études à temps partiel. Il s'intéresse enfin à l'apprentissage dans les environnements numériques et examine l'enseignement dans les nouveaux environnements d'apprentissage. http://bit.ly/2VdJZv8
\end{abstract}

Commission européenne, Report to the European Commission on new modes of learning and teaching in higher education, Office des publications de l'Union européenne/Luxembourg, 2014, 68p.

Ce rapport réalisé par le groupe de haut niveau de l'Union européenne (UE) sur la modernisation de l'enseignement supérieur présente des pistes d'action pour soutenir l'enseignement fondé sur les nouvelles technologies, sur la base d'une analyse approfondie de la situation actuelle et des bonnes pratiques constatées à travers l'UE et au-delà. Quinze recommandations mettent l'accent sur la nécessité de mieux cibler les ressources pour promouvoir le développement et l'utilisation de méthodes plus souples d'apprentissage et d'enseignement numériques dans l'enseignement supérieur.

EADTU : European association of distance teaching universities, HENDERIKX Piet et JANSEN Darco, The changing pedagogical landscape: in search of patterns in policies and practices of new modes of teaching and learning, EADTU/Maastricht, 2018, $114 p$. [en ligne]

Ce rapport soutient que les modes d'enseignement et d'apprentissage fondés sur les TIC peuvent résoudre les problèmes auxquels l'enseignement supérieur est confronté aujourd'hui. Il présente de nombreux exemples d'innovation pédagogique qui pourraient avoir un impact croissant les prochaines années. http://bit.ly/2IGWRmV

EADTU : European association of distance teaching universities, JANSEN Darco et KONINGS Lizzie, The 2018 OpenupEd trend report on MOOCs, EADTU/ Maastricht, 2018 [en ligne]

Les investissements dans les MOOCs (Massive open online courses) sont de plus en plus importants dans le monde entier. Coordonnée par OpenupEd, première initiative paneuropéenne 
de MOOCs, ce rapport présente les dernières innovations et développements en matière de MOOCs en Europe. Il réunit les contributions de 19 experts de douze organisations européennes. Les établissements d'enseignement supérieur européens sont fortement impliqués dans les MOOCs, utilisés à la fois pour offrir des opportunités d'apprentissage flexible et augmenter leur visibilité institutionnelle. http://bit.ly/2H3t3j5

EADTU : European association of distance teaching universities, UBACHS George ed., KONINGS Lizzie ed., The envisioning report for empowering universities: $2^{\text {nd }}$ edition, EADTU/Maastricht, 2018, 65 p., bibliogr. [en ligne]

Le programme EMPOWER, mis en œuvre par l'EADTU, soutient les établissements d'enseignement supérieur européens dans leur transition vers de nouvelles modalités d'enseignement et d'apprentissage. Assorti d'études de cas (Pays-Bas, Royaume-Uni, Irlande, Grèce, Espagne, Portugal, Turquie et Finlande), ce premier rapport du groupe d'experts du programme vise à présenter les derniers développements et tendances en matière d'apprentissage et d'enseignement tels que les learning analytics, l'apprentissage hybride, mais aussi les défis de l'université, le soutien aux étudiants et la formation continue. https://bit.ly/2s2R6WM

\section{ESU : European students union, Overview on student-centred learning in higher education in europe : research study, ESU/Bruxelles, 2015, 47 p. [en ligne]}

Ce rapport souligne les progrès effectués dans la mise en œuvre de "l'apprentissage centré sur l'étudiant " dans les établissements d'enseignement supérieur européens. Il recommande une plus grande consultation des étudiants dans l'élaboration des parcours et programmes d'études ainsi que dans les processus d'évaluation de contrôle des résultats d'apprentissage. Le 140 rapport préconise d'intégrer cette approche dans les stratégies, procédures et cadres institutionnels et de développer l'implication et la représentation des étudiants dans la gouvernance. http://bit.ly/2EjzCMI

EUA : European university association, BUNESCU Luisa, GAEBEL Michael, National initiatives in learning and teaching in Europe: a report from the European forum for enhanced collaboration in teaching (EFFECT) project, EUA/Bruxelles, décembre 2018, 29 p. [en ligne]

Réalisé dans le cadre du projet EFFECT, ce rapport analyse 28 systèmes d'enseignement supérieur afin de mieux comprendre comment la qualité de l'enseignement et de l'apprentissage s'est améliorée. Il souligne, entre autres, que le principal obstacle à l'amélioration de l'apprentissage et de l'enseignement est dû au fait que la recherche est davantage valorisée que l'enseignement, tant en termes de recrutement que de valorisation de la carrière des enseignants. https://bit.ly/2SrhJAm

\section{EUA : European university association, Promoting a European dimension to teaching enhancement : a feasibility study from the European Forum for Enhanced Collaboration in Teaching (EFFECT) projet, EUA/Bruxelles, 2019, 24 p. [en ligne]}

Le projet EFFECT a produit une étude de faisabilité sur la manière dont l'amélioration de l'enseignement peut être promue au niveau européen. Lancé lors du Forum européen sur l'apprentissage et l'enseignement de 2019, cette dernière présente et discute des approches pour le développement du personnel pédagogique déjà explorées dans les établissements et systèmes européens d'enseignement supérieur. Elle propose également des approches européennes pour soutenir et améliorer l'apprentissage et l'enseignement. http://bit.ly/2V7NvXV 
EUA : European University Association, GAEBEL Michael, ZHANG Thérèse, Trends 2018 : Learning and teaching in the European Higher Area, EUA/Bruxelles, octobre 2018, 104 p. [en ligne]

S'appuyant sur les réponses fournies par 303 établissements issus de 48 pays européens, ce rapport annuel de l'EUA présente les changements intervenus dans l'Espace européen de l'enseignement supérieur ces cinq dernières années. L'accent est mis sur les questions d'apprentissage et d'enseignement (stratégies institutionnelles, pilotage national, programmes d'études, méthodologies et approches pédagogiques, personnel enseignant). L'EUA constate que les établissements " adoptent progressivement des approches plus stratégiques et systématiques ». Elle observe d'autre part, que la carrière des personnels impliqués ne bénéficie pas ou peu des évaluations en enseignement. Ce manque de reconnaissance est identifié par les institutions comme l'« un des obstacles majeurs » pour améliorer l'enseignement et la pédagogie.

https://bit.ly/2GMuMLD

EUA : European university association, PAS Susan te et ZHANG Thérèse, Career paths in teaching: Thematic Peer Group Report, EUA/Bruxelles, 2019, 12 p. (Learning \& Teaching Paper, $n^{\circ}$ 2) [en ligne]

Ce rapport est le résultat d'un travail effectué par le Groupe thématique de pairs de l'EUA sur le parcours professionnel des enseignants. Il montre comment ce dernier peut contribuer à améliorer durablement l'enseignement supérieur en Europe, et il s'intéresse à ce qui peut être fait pour que la promotion de l'enseignement favorise la carrière des professeurs.

http://bit.ly/2XoOZKD

EUA : European university association, McINTYRE-BHATTY Tim et BUNESCU Luisa, Continuous development of teaching competences: thematic peer group report, EUA/Bruxelles, 2019, 12 p. (Learning \& Teaching Paper, $n^{\circ}$ 3) [en ligne]

À partir d'études de cas européens, ce rapport identifie différents défis en termes de formation initiale des enseignants et de développement professionnel : 1) les qualifications pédagogiques, les mécanismes de récompense et la motivation du personnel à poursuivre un développement professionnel continu ; 2) la mesure de l'impact du développement des compétences pédagogiques, dans le cycle d'assurance de la qualité et en lien avec l'innovation ; 3) le développement interdisciplinaire d'approches méthodologiques d'apprentissage et de pédagogie.

http://bit.ly/2Gs3aYr

EUA : European university association, DEWHIRST Claire et GOVER Anna, Evaluation of learning and teaching : Thematic Peer Group Report, EUA/Bruxelles, 2019, 10 p. (Learning \& Teaching Paper, $n^{\circ} 4$ ) [en ligne]

L'évaluation de l'apprentissage et de l'enseignement, principale activité par laquelle les universités contrôlent l'adéquation de leur offre éducative, a fait l'objet d'un groupe de travail de pairs de l'EUA. Ce document reflète les discussions du groupe sur les conditions cadres dans lesquelles les établissements assurent la qualité de leur enseignement. http://bit.ly/202uD7M

EUA : European university association, CHRISTERSSON Cecilia, STAAF Patricia, ZHANG Thérèse et PETERBAUER Hélène, Promoting active learning in universities: thematic peer group report, EUA/Bruxelles, 2019, 12 p. (Learning \& Teaching Paper, $n^{\circ} 5$ ) [en ligne] 
L'apprentissage actif englobe un large éventail d'approches pédagogiques impliquant l'apprenant à chaque étape du processus éducatif. Ce document est le résultat du travail effectué par le groupe de pairs thématique de l'EUA réuni autour de ce sujet. Il examine diverses approches de l'apprentissage actif et la façon dont elles peuvent être mises en œuvre. Il souligne la nécessité d'un changement culturel dans l'environnement académique, dans lequel les enseignants deviendraient des facilitateurs de l'apprentissage et les élèves leurs partenaires actifs. http://bit.ly/2PMw2zo

\section{AUtRes leViers POUR AMÉLIORER LA PÉDAGOGIE UNIVERSITAIRE : ÉTUDES DE CAS}

\section{Dispositifs et pratiques pédagogiques innovants}

DUMONT Ariane dir., BERTHIAUME Denis dir., La pédagogie inversée : enseigner autrement dans le supérieur avec la classe inversée, De Boeck supérieur/Louvain-laNeuve, 2016, 235 p.

Des auteurs américains, belges, canadiens, français et suisses traitent ici de la pédagogie inversée, décrivant les pratiques et/ou repères théoriques, historiques ou philosophiques dont ils s'inspirent pour mettre en ouvre des dispositifs s'apparentant à la classe inversée. La seconde partie de l'ouvrage présente des cas d'application de pédagogie inversée.

\section{LIM Cher Ping (ed.), WANG Libing (ed.), Blended learning for quality higher educa-} tion: selected case studies on implementation from Asia-Pacific, UNESCO Bureau régional pour l'éducation en Asie et dans le Pacifique/Bangkok, 2017, 296 p., [en ligne]

L'ouvrage explore le potentiel de l'apprentissage hybride dans la région Asie-Pacifique qui fait face à des demandes croissantes de modes plus souples d'accès à un enseignement supérieur de qualité. Il fournit un cadre et un outil d'auto-évaluation aux établissements d'enseignement supérieur. Des études de cas illustrent comment ce cadre peut être mis en œuvre et montrent pourquoi et comment les établissements adoptent une approche holistique afin de soutenir et stimuler l'apprentissage hybride au sein de leurs institutions respectives. http://bit.ly/2VcLOEu

POUMAY Marianne, TARDIF Jacques, GEORGES François (dir.), Organiser la formation à partir des compétences : un pari gagnant pour l'apprentissage dans le supérieur, De Boeck supérieur/Louvain la neuve, 2017, 364 p.

Cet ouvrage s'intéresse à l'organisation de formations professionnelles dans l'enseignement supérieur à partir des compétences essentielles au métier visé. Il s'agit de concevoir un programme axé sur le développement de compétences qui devra être transposé en formation dans les activités d'apprentissage et d'évaluation. Après avoir défini la notion de compétence et le champ théorique, les auteurs proposent un cadre méthodologique qui relie le programme et le développement de compétences et présentent onze études de cas (en France, au Liban, en Belgique et au Québec). 
RENSTA Bretagne, Telecom Bretagne, Université de Bretagne occidentale, Innover: pourquoi et comment? Actes du VIII colloque "Questions de pédagogies dans l'enseignement supérieur », Université de Bretagne occidentale/Brest, 2015, 949 p. [en ligne]

Ces actes proposent les retranscriptions de plus d'une centaine de communications, présentées lors du $8^{\mathrm{e}}$ colloque "Questions de pédagogies dans l'enseignement supérieur » consacré à l'innovation pédagogique, autant dans ses contenus (dispositifs innovants), que dans son environnement (politiques éducatives, démarches qualité) et dans ses finalités. Les communications s'appuient sur des études de cas en Amérique du Nord et en Europe. http://bit.ly/2WinRwR

SHARPLES Mike, ADAMS Anne, ALOZIE Nonye, et al., Innovating Pedagogy 2019: exploring new forms of teaching learning and assessment to guide educators and policy makers, The Open University/Milton Keynes, 2019, 45 p. [en ligne]

Réalisé en collaboration avec le Centre for the Science of Learning \& Technology (SLATE) de l'Université de Bergen, ce rapport est le septième d'une série sur les innovations dans l'enseignement, l'apprentissage et l'évaluation. Il présente dix innovations : apprendre en jouant, apprendre avec les robots, décoloniser l'apprentissage, l'apprentissage basé sur les drones, apprendre par l'émerveillement, apprendre par l'action, les studios virtuels, l'apprentissage basé sur l'environnement, rendre la pensée visible, et apprendre l'empathie. http://bit.ly/2WcYEqV

\section{Espaces physiques d'apprentissage}

ALBERO Brigitte, YURÈN Teresa; GUÉRIN Jérôme (éd.), Modèles de formation et architecture dans l'enseignement supérieur, Culture numérique et développement humain. Éditions Raison et Passions/Dijon, 2018, 359 p.

Cet ouvrage, qui mobilise seize auteurs mexicains et français, chercheurs en sciences humaines et sociales, enseignants, administrateurs et architectes, s'intéresse à ce que peuvent nous dire les bâtiments, les espaces, les modes de circulation et de vie et l'aménagement des lieux d'étude à propos de la façon dont l'institution fait face aux principaux défis de l'enseignement supérieur (massification, diversité des publics, généralisation du numérique, exigence de professionnalisation, etc.).

BÉRUBÉ Martin, "Learning center: innovation ou évolution des bibliothèques universitaires? ", Regroupement des bibliothèques collégiales du Québec, 6 février 2018 [en ligne]

Cet article revient sur le contexte qui a favorisé l'émergence du concept de "learning center ». Il tente de caractériser ce qui le différencie d'une bibliothèque universitaire classique et insiste notamment sur son offre de soutien à l'apprentissage. Enfin, il présente les changements provoqués par la mise en place de learning centers auprès de la communauté universitaire. http://bit.ly/2XTq2rq

BOSQUÉ Camille, NOOR Ophelia et RICARD Laurent, FabLabs, etc. Les nouveaux lieux de fabrication numérique, Eyrolles, 2014, 208 p. 
Au travers de retranscriptions d'interviews de différents acteurs français et internationaux liés aux FabLabs ou au mouvement Maker et Do It Yourself (DIY) en général, cet ouvrage fournit un état de l'art sur le sujet. Il s'intéresse notamment aux transformations que les FabLabs impliquent dans l'industrie, l'éducation et l'innovation.

GARRETTP. B, "The Evolving Classroom: Creating Experiential Learning Spaces ", EDUCAUSE Review, 13 octobre2014 [en ligne]

Cet article publié par Educause, association américaine à but non lucratif dont la mission est de promouvoir l'innovation dans l'enseignement supérieur via les nouvelles technologies, pointe le fait que l'apprentissage par l'expérience améliore l'engagement et l'apprentissage des étudiants et constitue ainsi un moteur pédagogique important dans la conception d'espaces d'apprentissages. http://bit.ly/2HOTJzJ

\section{Évaluation des compétences acquises et qualité des enseignements}

LIYANAGUNAWARDENA Tharindu R., SCALZAVARA Sandra et WILLIAMS Shirley A., "Open Badges: A Systematic Review of Peer-Reviewed Published Literature (2011-2015) ", European Journal of Open, Distance and E-Learning, vol. 20, $n^{\circ} 2$, 2017, p. 1-16 [en ligne]

Les badges ouverts (Open Badges) constituent un système standardisé de badges numériques qui permettent de certifier les compétences informelles. Ils ont gagné en popularité dans le monde entier ces dernières années et sont devenus une caractéristique de nombreux systèmes de gestion de l'apprentissage. Cet article présente une revue de la littérature publiée sur les badges ouverts de 2011 à 2015 et des suggestions pour les orientations futures de la recherche, en fonction des domaines sous-représentés. http://bit.ly/2HbAJ0Q

ROEGIERS Xavier, Collectif (collab.), Quelles réformes pédagogiques pour l'enseignement supérieur? L'intégration des acquis, une piste pour placer l'efficacité au service de l'humanisme, De Boeck/Bruxelles, 2012, 313 p.

L'ouvrage développe un ensemble de questions liées aux curricula dans l'enseignement supérieur : programmes d'études, organisation des apprentissages, évaluation des acquis des étudiants, supports de cours. La première partie replace la problématique dans une perspective large d'évolution des programmes dans les institutions d'enseignement supérieur et réinterroge le lien formation-emploi. La deuxième partie décrit l'approche par l'intégration des acquis de l'étudiant et sa mise en œuvre concrète dans les curricula.

\section{ROMAINVILLE Marc, GOASDOUÉ Rémi et VANTOUROUT Marc, Évaluation et enseignement supérieur, De Boeck/Bruxelles, 2013, 366 p.}

Cet ouvrage, qui s'appuie sur les contributions d'experts internationaux, s'intéresse à l'évaluation dans l'enseignement supérieur sous toutes ses formes : l'évaluation des acquis ; l'évaluation des enseignements, des formations et des programmes ; l'évaluation de la recherche et des chercheurs ; l'évaluation institutionnelle et de la qualité. Il met en avant les pratiques innovantes les plus porteuses ainsi que les rééquilibrages qu'il s'agirait d'opérer pour réconcilier les acteurs de l'enseignement supérieur avec l'évaluation. 
SOUTO LOPEZ Miguel, Acquis d'apprentissage et enseignement supérieur, Academia-L'Harmattan/Louvain-la-Neuve, 2016, 394 p.

Dans sa première partie, l'ouvrage retrace les origines de la notion de compétence dans l'enseignement aux États-Unis puis il s'intéresse à la diffusion de la notion de compétence à l'international. La seconde partie présente les divers instruments d'action publique construits soit par la Commission européenne, soit dans le cadre du processus de Bologne. La dernière partie de l'ouvrage cherche à comprendre ce qui a conduit à l'apparition de l'approche par compétences et à la généralisation de l'usage des acquis d'apprentissage dans l'enseignement supérieur belge francophone.

STRANG Lucy, BELANGER Julie, MANVILLE Catriona et al., Review of the research literature on defining and demonstrating quality teaching and impact in higher education, York: HEA : Higher education academy/York, 2016, 40 p. [en ligne]

Que signifient réellement " qualité », " excellence » et "impact », en matière d'enseignement ? Les débats sont vifs depuis des décennies et il n'existe pas de critères rigoureux permettant de mesurer et de démontrer la qualité de l'enseignement, contrairement à ceux qui sont à l'origine de l'évaluation de la recherche universitaire. En s'appuyant sur une revue de la littérature, cette étude vise à cartographier les concepts clés, les tendances et les impacts démontrables émergeant de ce débat, avec un accent particulier sur l'identification de "ce qui fonctionne". http://bit.ly/2LpdEOl

YOUNES Nathalie, PAIVANDI Saeed, DETROZ Pascal (coord.), "Pédagogie universitaire et évaluation de l'enseignement par les étudiants", Éducation et formation, $n^{\circ} e-307-1,2017,184 p$. [en ligne]

L'évaluation de l'enseignement par les étudiants (EEE) désigne les procédures par lesquelles ces derniers prennent part au jugement porté sur des enseignements. Cette démarche, devenue une norme institutionnelle dans un nombre croissant d'universités à travers le monde, vise avant tout à améliorer le fonctionnement et la qualité pédagogique de l'université. À partir d'expériences belges, françaises, libanaises et suisses, ce numéro s'intéresse à la conception et à l'évaluation de dispositifs d'EEE et à leurs effets. https://bit.ly/2wLbGwG

\section{Technologies éducatives}

BECKER ADAMS Samantha, BROWN Malcom, DAHLSTROM Eden et al., NMC Horizon report: 2018 higher education edition, EDUCAUSE/Louisville, 2018, 54 p. [en ligne]

Ce rapport annuel, synthèse de recherches et réflexions d'un panel d'experts en technologies éducatives venant de cinq continents vise, à partir d'exemples concrets, à identifier et à décrire les technologies émergentes susceptibles d'avoir un impact sur l'apprentissage, l'enseignement et la recherche créative. https://bit.ly/2wrocSo

BUGMANN Julien, JAILLET Alain et KARSENTI Thierry, "Certification universitaire post-MOOC : entre attentes et contraintes ", Revue internationale des technologies en pédagogie universitaire, 2018, vol. 15, $n^{\circ} 1$, p. 34-44 [en ligne] 
La question de la reconnaissance des certifications délivrées par les MOOC représente une sorte de plafond de verre qui limite leur développement. L'étude porte sur la caractérisation des différentes modalités d'évaluation avec une ambition de reconnaissance universitaire des MOOC, en se focalisant notamment sur les quelques initiatives qui proposent des solutions susceptibles d'apporter une validation institutionnelle à ce qui est mis en œuvre.

http://www.ijthe.org/fr/articles/view/321

KNYAZEVA Svetlana (ed.), Futures for higher education and ICT: changes due to the use of open content, UNESCO. IITE: Institute for information technologies in education/Moscow, 2016, 89 p., bibliogr. [en ligne]

Ce rapport fournit un panorama des tendances actuelles et futures liées à l'apprentissage ouvert et à distance dans l'enseignement supérieur. http://bit.ly/2Vdzyzf

\section{DÉVELOPPEMENT PROFESSIONNEL DES ENSEIGNANTS-CHERCHEURS}

BIÉMAR Sandrine, DAELE Amaury, MALENGREZ Déborah et OGER Laurence, "Le "Scholarship of Teaching and Learning" (SoTL). Proposition d'un cadre pour l'accompagnement des enseignants par les conseillers pédagogiques", Revue internationale de pédagogie de l'enseignement supérieur, juillet 2015, $n^{\circ} 312$ [en ligne]

Cet article présente le Scholarship of Teaching and Learning (SoTL), processus de développement de l'expertise des enseignants de l'enseignement supérieur en matière d'enseignement et d'apprentissage des étudiants. Les auteurs s'intéressent notamment aux étapes et aux processus du SoTL vécu par des enseignants de l'enseignement supérieur, ainsi qu'aux différentes postures de ces enseignants au regard de leur pratique et du développement de leur expertise en matière d'enseignement et d'apprentissage dans le supérieur. Plusieurs exemples issus d'une Haute École belge et d'une université suisse viennent illustrer les propos. http://ripes.revues.org/966

FRENAY Mariane et PAQUAY Léopold (coord.), "Former les universitaires en pédagogie » [dossier], Recherche \& formation, août 2012, vol. $n^{\circ} 67, n^{\circ} 2,175 p$. [en ligne]

Ce numéro rassemble les travaux de chercheurs venant principalement d'universités belges, britanniques, canadiennes et suisses. Ils présentent et analysent cinq dispositifs développés dans les universités pour permettre et soutenir le développement professionnel des enseignants-chercheurs. http://bit.ly/20oglzS

REGE COLET Nicole, BERTHIAUME Denis (ed.), La pédagogie de l'enseignement supérieur : repères théoriques et applications pratiques. Tome 2 : Se développer au titre d'enseignant, Peter Lang/Berne, 2015, 261 p.

À partir des contributions de conseillers pédagogiques, cet ouvrage explore treize dispositifs éprouvés contribuant au développement professionnel des enseignants parmi lesquels : les philosophies de l'enseignement, l'évaluation de l'enseignement auprès des étudiants, le mentorat, l'observation par les pairs, la pratique réflexive ou la recherche appliquée sur son enseignement. 
SECRETARY OF STATE FOR BUSINESS, INNOVATION AND SKILLS, Success as a Knowledge Economy: Teaching Excellence, Social Mobility and Student Choice, Department for Business, Innovation and Skills/Londres, mai 2016, 85 p. [en ligne]

Dans son livre blanc sur l'enseignement supérieur publié en mai 2016, le gouvernement britannique s'est engagé à mettre en place un nouveau système de reconnaissance de l'excellence dans l'enseignement supérieur. La loi de 2017 sur l'enseignement supérieur et la recherche (Higher education and research Act 2017) constitue la base législative du Teaching excellence and student outcomes framework (TEF), qui évalue la qualité des établissements d'enseignement supérieur (EES) en termes d'enseignement et garantit aux étudiants de bons résultats. http://bit.ly/2JSRXU6

\section{Recherche en éducation}

BRYK Anthony S., "Accélérer la manière dont nous apprenons à améliorer ", Éducation et didactique, 6 décembre 2017, vol. 11, $n^{\circ} 112$, p. 1129 [en ligne]

Dans cet article, Anthony S. Bryk, président de la Carnegie Foundation for the Advancement of Teaching à Stanford (Californie) préconise de faire de "l'amélioration » un secteur à part entière de la recherche en éducation et de développer des recherches de qualité sur les processus par lesquels un établissement peut améliorer ses effets sur les apprenants. Il soutient notamment l'idée de communautés d'amélioration en réseau (en anglais NIC: Networked Improvement Community). http://journals.openedition.org/educationdidactique/2796

GUEUDET Ghislaine, LAMEUL Geneviève et TROUCHE Luc, "Questions relatives à la "pédagogie universitaire numérique", regard et rôle de la recherche: Journées scientifiques "Pédagogie Universitaire Numérique ", Lyon, France, 8 (1/2), ", Revue Internationale des Technologies en Pédagogie Universitaire, 2011, p. 110 [en ligne]

Ce numéro présente des textes issus des premières journées scientifiques " Pédagogie universitaire numérique ", qui se sont tenues à Lyon (France) les 6 et 7 janvier 2011 sous l'égide de l'Institut français de l'éducation et de la Mission numérique pour l'enseignement supérieur. http://bit.ly/2EmVOjV

KARSENTI Thierry et SAVOIE-ZAJC Lorraine, La recherche en éducation : Étapes et approches (4e édition revue et mise à jour), PUM - Presse de l'université de Montréal. Enseigner et apprendre, 2018, 456 p.

Ce livre constitue une initiation générale aux méthodes de recherche en éducation. Il a pour but d'amener étudiants-chercheurs et praticiens à mieux comprendre les différentes étapes et les théories du processus de recherche en éducation. Cette quatrième édition comprend un nouveau chapitre sur la recherche ethnographique et un chapitre sur l'analyse des données qualitatives.

POTEAU Nicole, "De la recherche-action à la pédagogie universitaire: une démarche pour articuler enseignement et recherche ", Les dossiers des sciences de l'éducation, septembre 2015, $N^{\circ} 34, p .7590$ [en ligne] 
Après avoir présenté la démarche de recherche-action comme mode d'articulation entre recherche et pratique de terrain, en particulier dans sa valeur formative, l'article examine comment, dans l'enseignement supérieur français, amener les enseignants-chercheurs à établir des parallèles entre leurs activités d'enseignement et leurs activités de recherche.

\section{http://bit.ly/2VTWz3R}

REY Olivier, "Entre laboratoire et terrain : comment la recherche fait ses preuves en éducation : dossier ", Dossier de veille de l'IFÉ, $n^{\circ}$ 89, janvier 2014, 28 p. [en ligne]

Après avoir rappelé les enjeux de la situation actuelle de la recherche en éducation, le dossier fixe des repères pour une approche scientifique de l'éducation dans le contexte de la recherche universitaire. Puis, il revient sur la signification du concept controversé de l'evidence-based education (l'éducation basée sur les travaux de la recherche en éducation). Il envisage enfin comment peuvent être articulés les rapports entre recherche, pratiques et politiques éducatives, et l'engagement concret des acteurs qui relèvent de ces différents contextes. http://bit.ly/2HrJHJk 